\title{
Effets de la dose de gonadotrophines sur l'œestrus et la production d'embryons chez les brebis Hamra et Ouled Djellal
}

\author{
Ismail Gharbi ${ }^{1} *$ Amina Samia Dechicha ${ }^{1}$ Mustapha Ferrouk ${ }^{1}$ \\ Djamila Baazize-Ammi ${ }^{1}$ Seddik Kebbal ${ }^{1}$ Djamel Guetarni ${ }^{2}$
}

\section{Mots-clés}

Ovin, brebis Ouled Djellal, brebis Hamra, superovulation, pFSH, embryon animal, Algérie

Submitted: 1 April 2018

Accepted: 13 June 2018

Published: 29 October 2018

DOI: $10.19182 /$ remvt.31639

\section{Résumé}

L'application des techniques de superovulation et de production d'embryons est incontournable pour la conservation des ressources génétiques ovines. L'objectif de la présente étude a été d'évaluer l'effet de l'administration de deux doses d'hormone folliculostimulante d'origine porcine $(\mathrm{pFSH})$ sur le délai d'apparition et la durée de l'œestrus, la réponse ovarienne, et la production d'embryons chez les brebis des races Ouled Djellal (OD) et Hamra $(H)$. Les brebis OD $(n=15)$ et $H(n=14)$ ont été synchronisées par la pose d'éponges vaginales imprégnées avec $40 \mathrm{mg}$ d'acétate de fluorogestone (FGA), et superovulées par l'administration de 16 ou $20 \mathrm{UA}$ de pFSH durant les trois derniers jours du traitement progestagène. Le septième jour après œstrus et saillie naturelle, les embryons ont été collectés par laparotomie. Chez les brebis OD le début de l'œstrus a été plus précoce avec I'utilisation de 20 UA qu'avec celle de 16 UA $(22,1 \pm 4,5$ vs 25,0 $\pm 3,5, \mathrm{p}<0,05)$, de même la durée de l'oestrus a été plus longue 40,0 $\pm 7,1$ vs $34,0 \pm 6,6, p<0,05$. Chez les brebis $\mathrm{H}$ I'augmentation de la dose de $\mathrm{pFSH}$ de 16 UA à 20 UA a induit une baisse de production d'embryons : taux de collecte 72,2 vs 47,1, p < 0,001; nombre de structures récoltées 5,9 $\pm 3,4$ vs 3,6 $\pm 2,1, p<0,05$; nombre d'embryons récoltés $5,1 \pm 2,6$ vs 3,2 $\pm 1,9, p<0,05$; embryons de grade 1 et 2, 4,3 $\pm 2,4$ vs $2,1 \pm 1,7, p<0,01$. En revanche, chez les brebis OD le taux d'ovulation et la production d'embryons ont été nettement améliorés lors de I'utilisation d'une dose de 20 UA : nombre de corps jaunes $6,9 \pm 3,9$ vs $10,4 \pm 5,4, p<0,05$; nombre d'embryons récoltés $5,1 \pm 2,6$ vs 3,2 $\pm 1,9, p<0,05$; embryons de grade 1 et $2,2,2 \pm 1,6$ vs $4,3 \pm 1,7, p<0,05$. Les résultats indiquent que la réponse ovulatoire, l'œstrus, et la production d'embryons sont influencés par la dose de la pFSH et par la race.

- Pour citer cet article : Gharbi I., Dechicha A.S., Ferrouk M., Baazize-Ammi D., Kebbal S., Guetarni D., 2018. Effects of the gonadotropin dose on estrus and embryo production in Hamra and Ouled Djellal ewes. Rev. Elev. Med. Vet. Pays Trop., 71 (3) : 113-119, doi: 10.19182/remvt.31639

\section{INTRODUCTION}

En Algérie, Ouled Djellal (OD) et Hamra (H) comptent parmi les principales races ovines locales standardisées et normalisées (ITELV, 2012). Les ovins OD, plus importants numériquement (environ 11340000 têtes), représentent plus de $60 \%$ du cheptel national (Moula, 2018). Par ailleurs, les ovins Hamra ont vu leur effectif

\footnotetext{
1. Institut des sciences vétérinaires, Université de Blida 1, BP 270 Blida 09000, Algérie.

2. Faculté des sciences de la nature et de la vie, Université de Blida 1, Blida 09000 , Algérie.

* Auteur pour la correspondance

Tél. : +2130663687597 ; email : ism_guarb@yahoo.fr
}

diminuer drastiquement, pour passer de 2500000 têtes dans les années 1980 à 55800 têtes en 2003 (Feliachi et al., 2003). Les deux races ont connu des pratiques de croisements anarchiques ou non contrôlées (Djaout et al., 2017), situation qui favoriserait la disparition des standards phénotypiques, du potentiel génétique, et l'augmentation de la consanguinité dans les troupeaux. Les techniques de superovulation, de production et de transfert d'embryons sont d'un grand intérêt pour conserver les ressources génétiques ovines.

L'induction de la superovulation chez la brebis peut être réalisée par injection de gonadotrophine chorionique équine (eCG) ou d'extrait hypophysaire (hormones folliculostimulante / lutropine [FSH/LH]) (Gonzalez-Bulnes et al., 2004a). L'utilisation de la FSH permettrait une production plus importante d'embryons de qualité que celle de l'eCG (Oliveira et al., 2014 ; D'Alessandro et Martemucci, 2016). Chez les ovins, Baril et Guignot (2010) ainsi que Gibbons et Cueto (2011) 
rapportent que l'utilisation de la FSH dans le protocole « classique » de superovulation permet d'obtenir en moyenne 12 à 15 ovulations, avec des résultats pouvant varier de 0 à 20 . Cette grande variabilité dans la réponse à la superovulation demeure l'un des facteurs limitant la production d'embryons (Quan et al., 2011). En effet, elle est dépendante de nombreux facteurs intrinsèques comme l'âge, la race et le statut ovarien (Gonzalez-Bulnes et al., 2004a), et extrinsèques comme la saison, la nutrition, l'origine et la dose de gonadotrophine (Gonzalez-Bulnes et al., 2004a ; 2004b ; D’Alessandro et Martemucci, 2016).

Le facteur race représente approximativement $30 \%$ de la variabilité des rendements d'embryons obtenus en réponse au traitement FSH (Vivanco et al., 1994). Les différences de réponse superovulatoire entre les races sont liées au potentiel génétique des animaux (Baril et Guignot, 2010), à la prolificité différente des races, avec les meilleures réponses obtenues chez les races fortement prolifiques (Picazo et al., 1996 ; Dufour et al., 2000 ; Baril et Guignot, 2010). Néanmoins, des différences de rendement de superovulation ont été aussi trouvées en comparant des races non-prolifiques, où une interaction entre le type de gonadotrophine utilisé et la race a été décrite (Picazo et al., 1996). Ces différences entre races semblent être dues au recrutement et à la croissance d'un nombre élevé de follicules antraux présents dans l'ovaire au début du traitement FSH (Rebolledo et al., 2017), à des taux d'ovulation différents et à une fluctuation de la sécrétion de FSH et LH en réponse aux gonadotrophines exogènes (Ammoun et al., 2006).

La dose de gonadotrophines exogènes est un facteur clé pour l'obtention de réponses superovulatoires optimales et homogènes (Boscos et al., 1997). De nombreux travaux montrent qu'il existe un effet doseréponse de la FSH où les doses totales efficaces et recommandées chez la brebis sont comprises entre 16 et $20 \mathrm{mg}$ (Gibbons et Cueto, 2011 ; Oliveira et al., 2014). Elles peuvent cependant s'avérer insuffisantes ou au contraire excessives pour certains génotypes particuliers (Baril et Guignot, 2010). L'utilisation d'une faible dose de FSH diminuerait le nombre de follicules recrutés, le taux d'ovulation et par conséquent le nombre d'embryons transférables (Boscos et al., 1997 ; Gibbons et Cueto, 2011).

En revanche, l'administration d'une dose supérieure à 16 voire $20 \mathrm{mg}$ n'entraîne pas l'augmentation de la réponse ovulatoire (Gibbons et Cueto, 2011). Au contraire, elle peut provoquer une stimulation prolongée des follicules anovulatoires qui produisent des œstrogènes à des concentrations élevées et persistantes pendant la première phase lutéale, induisant ainsi la libération anticipée de prostaglandine F2 $\alpha$ (PGF2 $\alpha$ ) et la régression prématurée des corps jaunes (Maciel et al., 2017), phénomène aboutissant à la diminution du nombre d'embryons transférables (D’Alessandro et al., 1996 ; Maciel et al., 2017). De plus, quand plusieurs follicules sont stimulés par de fortes doses, les limitations physiques de l'ovaire, comme la diminution de l'apport sanguin aux follicules, le dérèglement des mécanismes endocriniens et la production excessive de stéroïdes ovariens, interfèrent avec le développement folliculaire ou l'ovulation (Katanya et al., 2009).

A notre connaissance, aucun essai de superovulation et de production d'embryons n'a concerné les races locales Hamra et Ouled Djellal. Ainsi cette étude proposait d'évaluer l'effet de l'administration de deux doses de FSH d'origine porcine ( $\mathrm{pFSH}$ ) sur les caractéristiques œstrales, la réponse ovarienne et la production d'embryons chez les brebis Ouled Djellal et Hamra.

\section{MATERIEL ET METHODES}

\section{Animaux et cadre de l'expérimentation}

L'étude a concerné des brebis et des béliers provenant des stations d'expérimentation de l'Institut technique des élevages (ITELV ; institution étatique de préservation des races pures) d'Ain M'Lila et de
Saïda, régions considérées comme les berceaux d'origine respectivement des races Ouled Djellal et Hamra. Les animaux ont été transportés et élevés dans un système semi-intensif (bergerie) sous éclairage naturel dans la ferme expérimentale de l'Université de Blidal située à 50 kilomètres d'Alger ( $36^{\circ} 28^{\prime} \mathrm{N}$ et $\left.2^{\circ} 49^{\prime} \mathrm{E}\right)$.

Les expérimentations ont été menées durant la période de reproduction (septembre à novembre). Elles ont concerné 29 brebis donneuses présentant un bon état sanitaire : 15 OD (5 primipares et 10 multipares) âgées de 2 à 5 ans et d'un poids vif variant entre 36 et $61 \mathrm{~kg}$; et $14 \mathrm{H}$ (4 primipares et 10 multipares) âgées de 2 à 6 ans et d'un poids vif variant entre 28 et $58 \mathrm{~kg}$. Quatorze béliers $(7 \mathrm{OD}$ et $7 \mathrm{H}$ ) reproducteurs âgés de 3 à 7 ans et d'un poids vif variant entre 52 et $78 \mathrm{~kg}$ ont été utilisés pour la détection des chaleurs et les saillies. Les animaux sélectionnés ont été déparasités un mois avant le début de l'étude avec de l'albendazole (Valbazen suspension, Pfizer Animal Health) et de l'ivermectine (Ivomec injectable, Merial, France). Leur régime alimentaire se composait de foin complémenté avec 500 grammes de concentré par animal par jour ; l'eau était distribuée ad libitum.

\section{Protocole hormonal}

Dans une étude croisée, toutes les brebis ont été soumises à deux protocoles de superovulation successifs $\left(\mathrm{T}_{1}\right.$ et $\left.\mathrm{T}_{2}\right)$ à deux mois d'intervalle (figure 1). L'œstrus a été induit par le retrait, après 14 jours, d'une éponge vaginale imprégnée de $40 \mathrm{mg}$ d'acétate de fluorogestone (FGA) (Chronogest, Intervet, France). Chaque brebis a été superovulée par l'administration successive de 16 ou 20 unités Armour (UA) de pFSH purifiée (Stimufol, Reprobiol, Université de Liège, Belgique). La pFSH (160 $\mu \mathrm{g}$ ou $200 \mu \mathrm{g}$; une unité de $1 \mathrm{mg}$ $\mathrm{UA}=10 \mu \mathrm{g}$ de FSH pure) a été administrée à doses décroissantes en six injections réalisées matin et soir au cours des trois derniers jours du traitement par le progestagène. Les deux dernières injections de pFSH ont été enrichies par addition respectivement de $60 \mu \mathrm{g}$ et $90 \mu \mathrm{g}$ de $\mathrm{pLH}(\mathrm{FSH} / \mathrm{LH}=0,4$ et 0,3$)$. Une injection intramusculaire de 125 mg de cloprosténol (Estrumate : analogue de $\mathrm{PGF}_{2} \alpha$, solution injectable, Intervet) a été réalisée le jour et le lendemain de la récolte des embryons afin d'éviter une gestation.

\section{Détection de l'oestrus et saillie}

La détection de l'œstrus a été réalisée à l'aide de béliers entiers munis d'un tablier toutes les quatre heures, de la $12^{\mathrm{e}}$ à la $96^{\mathrm{e}}$ heure après le retrait de l'éponge vaginale. Toute donneuse restant immobile lors du chevauchement par le bélier détecteur a été considérée en chaleurs et mise en contact avec le bélier reproducteur. Une double saillie en main a été aussi réalisée, à 32 et 44 heures après le retrait de l'éponge vaginale avec des béliers reproducteurs (de la race respective) à raison d'un mâle pour cinq brebis.

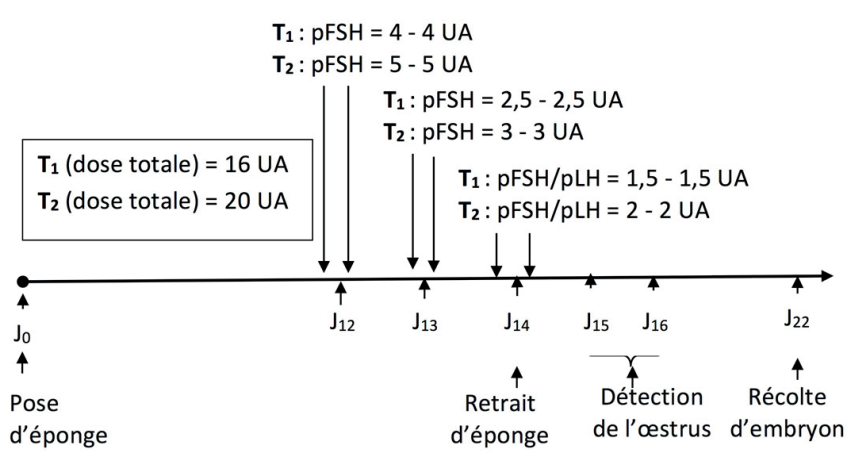

Figure 1 : protocoles de traitements de synchronisation et de superovulation des brebis de race Hamra et Ouled Djellal à la ferme expérimentale de l'Université de Blida en Algérie. 


\section{Dénombrement des corps jaunes, récolte, recherche et classification des embryons}

Pour éviter de récolter chirurgicalement les femelles présentant un nombre de corps jaunes inférieur à 2, la réponse ovarienne a été évaluée par laparoscopie sept jours après le début de l'œstrus (Baril et al., 1993), par le dénombrement des corps jaunes et des follicules anovulatoires. Les embryons ont été récoltés par laparotomie médioventrale selon le protocole conventionnel utilisé chez les petits ruminants (Baril et al., 1993). La laparoscopie et la laparotomie ont été effectuées sous anesthésie générale par l'administration intramusculaire de $0,2 \mathrm{mg} / \mathrm{kg}$ de poids vif de xylazine (Rompun Bayer Ag, Leverkusen, Germany) et de $5 \mathrm{mg} / \mathrm{kg}$ de poids vif de kétamine (Imalgène1000, Mérial, Lyon, France).

Les embryons ont été récoltés par lavage successif rétrograde des deux cornes (Baril et al., 1993). Il consiste à injecter, au niveau de la base de la corne utérine, $40 \mathrm{ml}$ de tampon PBS (phosphate-buffered saline ; IMV Technologies, L'Aigle, France) supplémenté de 4\%o de BSA (bovine serum albumine; IMV Technologies). La récupération du milieu de récolte a été réalisée à l'extrémité opposée de la corne, près de la jonction utérotubaire par ponction et insertion d'un cathéter de collecte muni d'une aiguille de perfusion émoussée (figure 2).

Après filtration et décantation du milieu de récolte, une recherche et un classement des embryons selon les critères morphologiques décrits par Robertson et Nelson (1999) ont été réalisés sous loupe binoculaire et microscope inversé (x 20 à 400). Les embryons ont été classés selon les critères morphologiques de l'International Embryo Transfer Society (IETS ; Robertson et Nelson, 1999), à savoir :
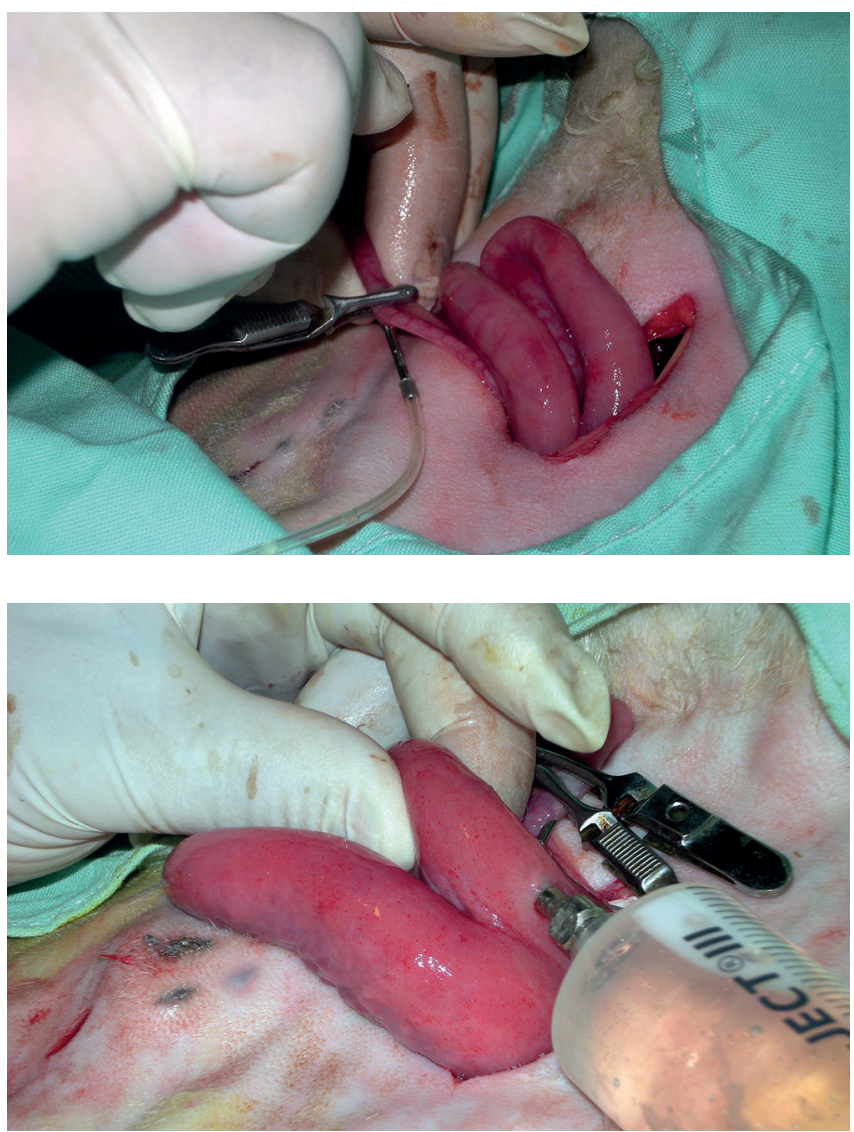

Figure 2 : méthode de collecte rétrograde des embryons à la ferme expérimentale de l'Université de Blida en Algérie; en haut : mise en place d'un cathéter de collecte au niveau de la jonction utéro-tubaire; en bas : injection de PBS au niveau de la base de la corne utérine. (C) I. Gharbi)
- grade 1, excellent (embryons congelables) ; l'embryon est symétrique et sphérique ; les blastomères sont uniformes en taille, couleur et densité ; le stade de développement est en adéquation avec la date de récolte ; au moins $85 \%$ des cellules sont intactes et rattachées à la masse cellulaire ; la zone pellucide est lisse et sphérique ;

- grade 2, bon ou satisfaisant (embryons transférables mais noncongelables); des irrégularités modérées concernent la forme globale de l'embryon, la taille, la couleur ou la densité de ses cellules ; au moins $50 \%$ de sa masse cellulaire est intacte ;

- grade 3, médiocre (embryons non-transférables et non-congelables) ; des irrégularités majeures concernent la forme de l'embryon, la taille, la couleur ou la densité de ses cellules ; au moins $25 \%$ de sa masse cellulaire est intacte ;

- grade 4, développement arrêté (embryon mort ou dégénéré).

\section{Paramètres évalués et analyses statistiques}

Les paramètres suivants ont été évalués : le délai d'apparition de l'œstrus (intervalle de temps compris entre le retrait de l'éponge vaginale et la première acceptation du chevauchement), la durée de l'œstrus (intervalle de temps compris entre la première et la dernière acceptation du chevauchement), le pourcentage de brebis en œstrus (nombre [nb.] de brebis détectées en œstrus / nb. total de brebis traitées x 100), le pourcentage de récolte (nb. d'ovocytes non-fécondés et d'embryons récoltés / nb. total de corps jaunes x 100), le pourcentage de fécondation (nb. d'embryons récoltés / nb. total d'ovocytes non-fécondés et d'embryons récoltés $x$ 100), et le pourcentage d'embryons de grade 1 et 2 (nb. d'embryons de grade 1 et $2 / \mathrm{nb}$. total d'embryons x 100).

Les données relatives à l'œstrus (début et durée de l'œstrus), à la qualité des embryons (grade 1,2,3 et 4), aux ovocytes non-fécondés et aux embryons récoltés ont été soumises à une analyse de la variance (Anova). Le test t de Student a été utilisé pour comparer l'effet de la race et du traitement sur la réponse ovarienne (corps jaunes, follicules anovulatoires). Les pourcentages des femelles en œstrus, de collecte, de fécondation et d'embryons de grade 1 et 2 par traitement, ont été analysés en utilisant le test du Chi carré. Le logiciel SYSTAT (version 10) a été utilisé pour toutes les analyses statistiques (niveau de signification $\mathrm{p} \leq 0,05)$.

\section{RESULTATS}

\section{Effet de la dose de pFSH sur le comportement ostral, la réponse ovarienne et la production d'embryons}

L'œstrus a été détecté chez la totalité des brebis OD et $\mathrm{H}$ de 16 à 32 heures (soit en moyenne 23,2 $\pm 3,8 \mathrm{~h}$ ) après le retrait de l'éponge vaginale. Sa durée a été de 36,1 \pm 7,2 h (figure 3).

Chez les brebis OD traitées avec 20 UA l'oestrus a été plus précoce qu'avec $16 \mathrm{UA}(22,1 \pm 4,5 \mathrm{~h}$ vs $25,0 \pm 3,5 \mathrm{~h}, \mathrm{p}=0,05)$; de même la durée moyenne de l'œstrus a été significativement plus longue $(40,0$ $\pm 7,1 \mathrm{~h}$ vs $34,0 \pm 6,6 \mathrm{~h}, \mathrm{p}=0,02$ ) et un plus grand nombre de corps jaunes a été observé $(10,4 \pm 5,4$ vs $6,9 \pm 3,9, p=0,05)$ (tableau I). En outre, le nombre moyen de structures récoltées a eu tendance à être plus élevé à la dose de 20 UA $(6,1 \pm 3,7$ vs $4,0 \pm 2,3, p=0,07)$ et le nombre moyen d'embryons récoltés a été significativement plus élevé $(5,1 \pm 2,3$ vs $3,2 \pm 2,0, p=0,02)$. Aucune différence significative n'a été observée entre les deux traitements pour le taux de collecte $(60,1 \%$ vs $58,6 \%, p=0,81)$ et de fécondation $(83,7 \%$ vs $80,3 \%$, $\mathrm{p}=0,59)$. L'appréciation de la qualité des embryons a révélé que chez les brebis OD le nombre moyen d'embryons de grade 1 et 2 a été significativement ( $\mathrm{p}=0,01$ et $\mathrm{p}=0,006$ ) plus élevé avec $20 \mathrm{UA}$ qu'avec 16 UA. Concernant le nombre moyen d'embryons de grade 3 et 4 , aucune différence significative n'a été observée entre les deux traitements (tableau II). 


\section{Tableau I}

Effet de la dose de pFSH et de la race ovine sur les caractéristiques de l'œstrus et la réponse ovarienne à la ferme expérimentale de I'Université de Blida en Algérie

\begin{tabular}{|c|c|c|c|c|}
\hline & \multicolumn{2}{|c|}{ Ouled Djellal } & \multicolumn{2}{|c|}{ Hamra } \\
\hline & \multicolumn{2}{|c|}{ Dose de pFSH } & \multicolumn{2}{|c|}{ Dose de pFSH } \\
\hline & 16 UA & 20 UA & 16 UA & 20 UA \\
\hline Brebis traitées & 15 & 15 & 14 & 14 \\
\hline Brebis en œestrus & $15 / 15$ & $15 / 15$ & $14 / 14$ & $14 / 14$ \\
\hline $\begin{array}{l}\text { Début de } \\
\text { l'œstrus }^{1}(h)\end{array}$ & $22,1 \pm 4,5^{b}$ & $25,0 \pm 3,5^{a}$ & $23,1 \pm 2,3$ & $22,6 \pm 4,3$ \\
\hline $\begin{array}{l}\text { Durée de } \\
\text { l'œstrus }^{1}(h)\end{array}$ & $34,0 \pm 6,6^{a}$ & $40,0 \pm 7,1^{b}$ & $34,6 \pm 6,2$ & $35,7 \pm 8,1$ \\
\hline Corps jaunes 1 & $6,9 \pm 3,9^{a}$ & $10,4 \pm 5,4^{b}$ & $8,5 \pm 3,5$ & $7,4 \pm 3,7$ \\
\hline $\begin{array}{l}\text { Follicules } \\
\text { anovulatoires }{ }^{1} \\
(\geq 4 \mathrm{~mm})\end{array}$ & 0 & $0,1 \pm 0,2$ & $0,1 \pm 0,3$ & 0 \\
\hline
\end{tabular}

${ }^{1}$ Moyenne \pm écart type. Les valeurs présentant sur une même ligne des lettres en exposant sont significativement différentes $\left({ }^{\mathrm{a}} \mathrm{vs}^{\mathrm{b}}, \mathrm{p} \leq 0,05\right)$.

Chez les brebis $\mathrm{H}$ aucune différence significative n'a été observée entre les deux traitements pour le délai d'apparition de l'œstrus (environ $23 \mathrm{~h}, \mathrm{p}=0,66$ ) et la durée de l'œstrus (environ $35 \mathrm{~h}, \mathrm{p}=0,67$ ). Le traitement avec 20 UA a induit un nombre moins élevé de corps jaunes qu'avec 16 UA $(7,4 \pm 3,7$ vs $8,5 \pm 3,5, p=0,44)$ (tableau II). En outre, l'augmentation de la dose d'hormone de 16 UA à 20 UA a eu un effet significativement négatif sur le taux de collecte (72,2 vs $47,1, \mathrm{p}<0,001)$, le nombre moyen de structures récoltées $(5,9 \pm 3,4$ vs $3,6 \pm 2,1, p=0,03)$ et le nombre d'embryons récoltés $(5,1 \pm 2,6$ vs 3,2 $\pm 1,9, p=0,04)$ de grade $1(p=0,01)$ et de grade $2(p=0,03)$. Aucune différence significative n'a été observée entre les deux traitements pour le nombre moyen d'embryons de grade 3 et 4 (tableau II).

\section{Effet de la race sur le comportement oestral, la réponse ovarienne et la production d'embryons}

A la dose de 16 UA l'œstrus est apparu plus précocement $(\mathrm{p}=0,09)$ chez les brebis $\mathrm{H}(23,1 \pm 2,3 \mathrm{~h})$ que chez les brebis OD $(25,0 \pm 3,5 \mathrm{~h})$. Aucune différence significative $(\mathrm{p}=0,26)$ n'a été observée entre les deux races pour la réponse ovulatoire. Néanmoins, chez les brebis $\mathrm{H}$ le rendement de la production d'embryons a été significativement plus élevé que chez les brebis OD avec un taux de collecte de 72,2 vs $58,6(\mathrm{p}=0,03)$, un nombre moyen d'embryons récoltés de 5,1 $\pm 2,6$ vs $3,2 \pm 2,0(p=0,03)$, de grade 1 de $3,2 \pm 2,1$ vs $1,7 \pm 1,0(p=0,02)$, de grade 2 de $1,1 \pm 0,8$ vs $0,5 \pm 0,8$ ( $p=0,05)$, et un taux d'embryons de grade 1 et 2 de $85,9 \%$ vs $69,4 \%(p=0,02)$ (tableau II).

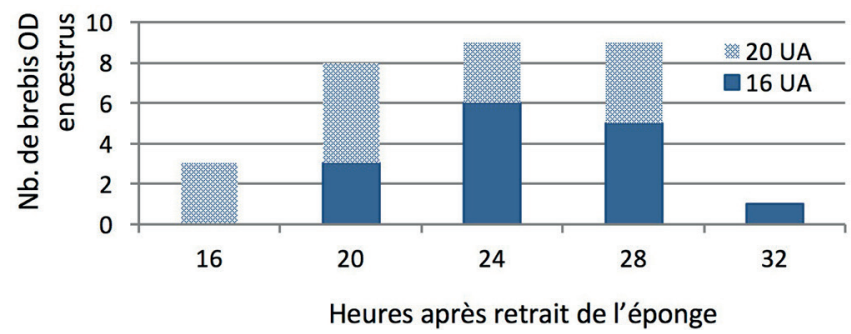

\section{Tableau II}

Effet de la dose de pFSH (16 UA, 20 UA) et de la race ovine sur la production d'embryons à la ferme expérimentale de I'Université de Blida en Algérie

\begin{tabular}{|c|c|c|c|c|}
\hline & \multirow{2}{*}{\multicolumn{2}{|c|}{$\begin{array}{l}\text { Ouled Djellal } \\
\text { Dose de pFSH }\end{array}$}} & \multirow{2}{*}{\multicolumn{2}{|c|}{$\begin{array}{c}\text { Hamra } \\
\text { Dose de pFSH }\end{array}$}} \\
\hline & & & & \\
\hline & $16 \mathrm{UA}$ & 20 UA & $16 \mathrm{UA}$ & $20 \mathrm{UA}$ \\
\hline Brebis récoltées & 15 & 15 & 14 & 14 \\
\hline $\begin{array}{l}\text { Structures } \\
\text { récoltées }{ }^{1}\end{array}$ & $4,0 \pm 2,3$ & $6,1 \pm 3,7^{a}$ & $5,9 \pm 3,4^{\mathrm{a}}$ & $3,6 \pm 2,1^{b}$ \\
\hline $\begin{array}{l}\text { Taux de } \\
\text { récolte (\%) }\end{array}$ & $58,6^{\mathrm{a}}$ & $60,1^{\mathrm{a}}$ & $72,2^{\mathrm{C}}$ & $47,1^{d}$ \\
\hline Embryons ${ }^{1}$ & $3,2 \pm 2,0^{\mathrm{a}}$ & $5,1 \pm 2,3^{b}$ & $5,1 \pm 2.6^{b}$ & $3,2 \pm 1,9^{a}$ \\
\hline $\begin{array}{l}\text { Ovocytes } \\
\text { non fécondés } 1\end{array}$ & $0,8 \pm 0,9$ & $1,0 \pm 1,4$ & $0,8 \pm 1,2$ & $0,4 \pm 0,5$ \\
\hline $\begin{array}{l}\text { Taux de } \\
\text { fécondation (\%) }\end{array}$ & 80,3 & 83,7 & 85,5 & 89,8 \\
\hline \multicolumn{5}{|l|}{$\begin{array}{l}\text { Qualité des } \\
\text { embryons }{ }^{1}\end{array}$} \\
\hline Grade 1 & $1,7 \pm 1,0^{\mathrm{a}, \mathrm{e}}$ & $3,0 \pm 1,6^{f}$ & $3,2 \pm 2,1^{b, f}$ & $1,6 \pm 1,2^{\mathrm{e}}$ \\
\hline Grade 2 & $0,5 \pm 0,8^{\mathrm{a}, \mathrm{e}}$ & $1,3 \pm 0,6^{f}$ & $1,1 \pm 0,8^{b}$ & $0,5 \pm 0,8^{c, e}$ \\
\hline Grade 3 & $0,5 \pm 0,6$ & $0,1 \pm 0,3$ & $0,1 \pm 0,3$ & $0,2 \pm 0,5$ \\
\hline Grade 4 & $0,5 \pm 0,6$ & $0,7 \pm 0,7$ & $0,7 \pm 0,9$ & $0,9 \pm 0,8$ \\
\hline $\begin{array}{l}\text { Embryons de } \\
\text { grade (1 et } 2)^{1}\end{array}$ & $2,2 \pm 1,6^{\mathrm{e}}$ & $4,3 \pm 1,7^{f}$ & $4,3 \pm 2,4^{f}$ & $2,1 \pm 1,7^{e}$ \\
\hline $\begin{array}{l}\text { Taux } \\
\text { d'embryons de } \\
\text { grade (1 et } 2 \text { ) (\%) }\end{array}$ & $\begin{array}{c}34 / 49 \\
(69,4)^{a}\end{array}$ & $\begin{array}{c}65 / 77 \\
(84,4)^{\mathrm{b}, \mathrm{e}}\end{array}$ & $\begin{array}{c}61 / 71 \\
(85,9)^{\mathrm{b}, \mathrm{e}}\end{array}$ & $\begin{array}{l}29 / 44 \\
(65,9)^{f}\end{array}$ \\
\hline
\end{tabular}

${ }^{1}$ Moyenne \pm écart type. Les valeurs présentant sur une même ligne des lettres en exposant sont significativement différentes $\left({ }^{\mathrm{a}} \mathrm{vs}^{\mathrm{b}},{ }^{\mathrm{a}} \mathrm{vs}{ }^{\mathrm{c}},{ }^{\mathrm{a}} \mathrm{vs}{ }^{\mathrm{d}},{ }^{\mathrm{b}} \mathrm{vs}{ }^{\mathrm{c}}, \mathrm{p} \leq 0,05 ;{ }^{\mathrm{e}} \mathrm{vs}\right.$ ${ }^{\mathrm{f}}, \mathrm{p}<0,01 ;{ }^{\mathrm{c}}$ vs $\left.{ }^{\mathrm{d}}, \mathrm{p}<0,001\right)$.

A la dose de 20 UA, la durée de l'œstrus tendait à être plus longue chez les brebis OD $(40,0 \pm 7,1)$ que chez les brebis $\mathrm{H}(35,7 \pm 8,1)(\mathrm{p}=0,10)$. De plus, le nombre moyen de corps jaunes chez les brebis OD tendait à être significativement plus élevé $(10,4 \pm 5,4$ vs $7,4 \pm 3,7, p=0,09)$. Un effet significatif de la race sur le rendement de la superovulation a aussi été mis en évidence. Chez les brebis OD le taux de collecte (60,1 vs $47,1, p=0,04)$, le nombre moyen de structures récoltées $(6,1 \pm 3,7$ vs 3,6 $\pm 2,1, \mathrm{p}=0,02)$, d'embryons récoltés $(5,1 \pm 2,3$ vs $3,2 \pm 1,9, \mathrm{p}=0,02)$, de grade $1(3,0 \pm 1,6$ vs $1,6 \pm 1,2, p=0,01)$, de grade $2(1,3 \pm 0,6$ vs 0,5 $\pm 0,8, \mathrm{p}=0,003)$, et le taux d'embryons de grade 1 et $2(84,4$ vs 65,9 , $\mathrm{p}=0,01)$ ont été significativement plus élevés que chez les brebis $\mathrm{H}$.

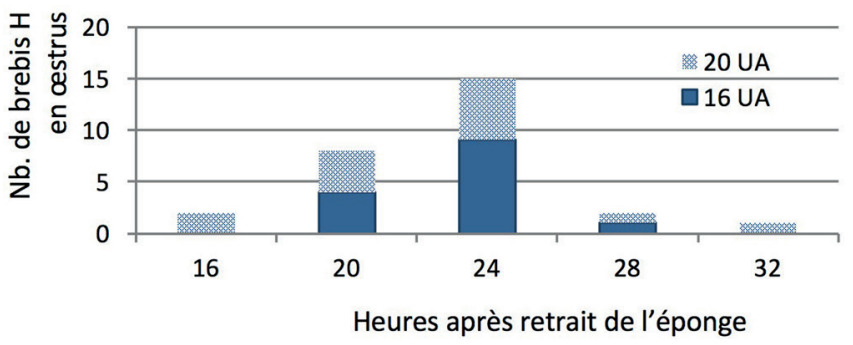

Figure 3 : début de l'œestrus chez les brebis Ouled Djellal (OD) et Hamra (H) traitées avec 16 UA et 20 UA de pFSH à la ferme expérimentale de I'Université de Blida en Algérie. 


\section{DISCUSSION}

Cette étude a confirmé l'effet significatif de la dose de pFSH et de la race sur la réponse à un traitement de superovulation. Il est à noter que la comparaison des deux doses de pFSH chez les deux races a été réalisée au moyen d'un protocole expérimental contrôlé et des conditions standardisées. Le choix de ce protocole sous forme d'étude croisée a révélé l'effet du traitement pFSH et de la race et ainsi d'accroître le potentiel de notre étude. En effet, ce type de méthode où chaque brebis est son propre témoin permet de réduire le nombre de sujets nécessaires (essais de petits effectifs), d'améliorer la fiabilité des résultats et est particulièrement indiqué si la variabilité de la réponse interindividuelle est importante (Vray et al., 2004).

Nos protocoles de superovulation ont induit l'apparition d'un œstrus chez $100 \%$ des brebis des deux races. L'intervalle moyen entre le retrait de l'éponge et le début de l'œstrus, compris entre 16 et 32 heures, a ainsi été comparable à celui rapporté par Baril et al. (1993) chez des brebis Lacaunes traitées avec 20 UA. Le recours à une dose de 20 UA a contribué chez la race OD à induire l'apparition plus précoce et plus longue de l'œstrus. Cette observation a été rapportée également par d'autres auteurs (Baril et al., 1993 ; D'Alessandro et Martemucci, 2004). Dans la présente étude, les brebis n'ont pas reçu d'injection de $\mathrm{PGF}_{2} \alpha$ en fin de traitement de superovulation pour contrôler le cycle œstral. En effet, chez la brebis, l'effet des prostaglandines $\left(\mathrm{PGF}_{2} \alpha\right.$ ou ses analogues synthétiques) dans les protocoles de traitements de synchronisation et de superovulation a été étudié, mais les conclusions sont contradictoires et leur rôle n'a pas clairement été établi. Il semble que l'utilisation de PG altère le transport des spermatozoïdes et diminue le taux de gravidité et de récolte des embryons (Fierro et al., 2013). De plus, selon ces auteurs, l'altération du taux d'ovulation et de la capacité stéroïdogénique des follicules préovulatoires, la qualité des embryons, et la prolificité après l'administration de PG sont controversées.

Chez les brebis OD le nombre de corps jaunes a augmenté avec l'augmentation de la dose de pFSH. A la dose de $20 \mathrm{UA}$, le nombre de corps jaunes a été comparable à celui obtenu par Lopez-Junior et al. (2006) chez des brebis Morada Nova $(10,2 \pm 1,2)$, mais inférieur à celui rapporté par Baril et Guignot (2010) chez les brebis Black Belly $(13,9 \pm 4,9)$. A l'inverse chez les brebis $\mathrm{H}$, le nombre de corps jaunes a diminué avec l'augmentation de la dose. Cet effet de la dose a également été rapporté par Buckrell et al. (1990) chez la race Dall's (Ovis dalli dalli). Le nombre de corps jaunes obtenus après injection de 16 UA de pFSH a été similaire à celui rapporté par Chagas e Silva et al. (2003) chez les brebis Saloia, mais plus faible que celui observé par Boscos et al. (1997) chez les brebis Chios. Il semblerait donc utile de pouvoir déterminer la dose optimale d'un traitement de superovulation au moyen d'une courbe dose-réponse (Baril et al., 1993). Selon Kanitz et al. (2002), l'utilisation de doses croissantes de FSH permet une augmentation significative du nombre d'ovulations jusqu'à l'obtention d'un plateau. Toutefois l'augmentation de la dose n'induit pas nécessairement celle du nombre d'ovulations.

Selon nos observations, les doses de 16 UA et de 20 UA de pFSH semblent devoir être réservées respectivement aux brebis H et OD. Il y aurait donc bien une sensibilité différente des races à la dose injectée (González et al., 2001). Cette observation a également été rapportée chez d'autres races de brebis et chez la chèvre. Avec le même traitement pFSH, le nombre moyen d'ovulations est inférieur chez les chèvres Alpines par rapport à celui observé chez les chèvres Créoles (Baril et Guignot, 2010). La réponse ovulatoire des brebis Lacaunes est inférieure à celle des brebis prolifiques Romanov x Préalpes lorsqu'elles sont traitées avec une dose de $16 \mathrm{mg}$ de pFSH (Torres et Cognié, 1984). Pour une dose de 20 UA, les réponses sont très élevées chez la Booroola et très faibles chez la Mérinos de Rambouillet (Baril et Guignot, 2010). Dans notre étude, l'efficacité moindre du traitement pFSH observée chez la race $\mathrm{H}$ par rapport à la race $\mathrm{OD}$ était peut-être due à une population folliculaire différente (Cognié et al., 2003 ; Gonzalez-Bulnes et al., 2004b ; Baril et Guignot, 2010), en lien avec sa prolificité (Picazo et al., 1996 ; Boscos et al., 1997), ou encore son degré de consanguinité (Baril et Guignot, 2010). Il n'est pas exclu que cette réponse différente soit liée à un comportement cinétique différent des gonadotrophines exogènes, à une dynamique folliculaire différente ou encore à une sensibilité plus élevée de récepteurs ovariens à la FSH (Dufour et al., 2000). De plus, selon Ammoun et al. (2006) et Rahman et al. (2014) la différence de poids qui existe entre les races peut aussi influencer les mécanismes de distribution et d'élimination des gonadotrophines.

L'administration de doses décroissantes de pFSH permet l'obtention d'une réponse ovulatoire plus élevée que celle à base de doses constantes (Cognié et al., 2003 ; Gonzalez-Bulnes et al., 2004a), et un apport croissant de $\mathrm{LH}$ en fin de traitement améliore la réponse ovulatoire (Cognié et al., 2003 ; D’Alessandro et al., 2005). L'apport de $\mathrm{pLH}$ décalé par rapport à la FSH semble correspondre à l'environnement hormonal physiologique du follicule en croissance terminale (Cognié et al., 2003). Une faible incidence des follicules anovulatoires a été observée chez les deux races. Il est à noter que, comparé à la gonadotrophine chorionique équine (eCG), le traitement à base de FSH/LH permet de réduire l'incidence des follicules anovulatoires et l'hypertrophie des ovaires (Martemucci et al., 1997, Cognié, 1999), et d'obtenir un taux élevé et synchrone des ovulations (Cognié, 1999).

Les pourcentages de récolte d'embryons obtenus par voie chirurgicale chez les races OD et $\mathrm{H}$ après traitement avec 16 ou 20 UA ont été comparables à ceux rapportés par Gonzalez Bulnes et al. (2004a), Ammoun et al. (2006), et Lopez -Junior et al. (2006) (50 à $75 \%$ chez les races Manchega ou Morada Nova. La voie chirurgicale semble donc bien devoir être privilégiée par rapport à la voie cervicale ou laparoscopique (Baril et al., 1993 ; Gibbons et Cueto, 2011). La diminution du nombre d'embryons récoltés semble être due à l'effet inhibiteur exercé par le ou les follicules dominants présents au début d'un traitement de superovulation (Baril et Guignot, 2010 ; Gonzalez-Bulnes et al., 2002 ; Veiga-Lopez et al., 2006).

Les pourcentages de fertilisation observés chez les races OD et $\mathrm{H}$ ont été légèrement inférieurs à ceux de plus de $90 \%$ rapportés par Bari et al. (2003). Cette réduction serait imputable aux effets hormonaux indirects induits par la superovulation sur la synthèse des œstrogènes et donc la libération de l'hormone LH (Cognié et al., 2003 ; Gonzalez Bulnes et al., 2004a). Le nombre moyen et le pourcentage d'embryons de grade 1 et 2 obtenus dans la présente étude ont été plus élevés après injection de $20 \mathrm{UA}$ et $16 \mathrm{UA}$ respectivement chez les brebis OD et $\mathrm{H}$. Ces constatations confirment que, chez la brebis, le nombre et la qualité d'embryons transférables sont aussi dépendants de la race (Baril et Guignot, 2010). En utilisant la même dose de 20 UA de FSH, Guignot et al. (2009), et Baril et Guignot (2010) rapportent des taux d'embryons transférables faibles et comparables $(2,4 \pm 2,5)$, respectivement chez les races Ile de France et Mérinos de Rambouillet. En revanche, un nombre élevé d'embryons transférables $(22,8 \pm 9,9)$ a été obtenu par Baril et Guignot (2010) chez la Booroola. Le nombre élevé d'embryons de très bonne qualité obtenu chez certaines races semble être la conséquence d'une forte réponse ovulatoire au traitement FSH (Cognié et al., 2003 ; Baril et Guignot, 2010).

\section{CONCLUSION}

Cette étude confirme les effets de la race et de la dose de pFSH sur les caractéristiques œstrales, la réponse ovarienne et le nombre d'embryons obtenus. La dose de pFSH semble devoir être adaptée à la race. Les contraintes liées à la production d'embryons chez l'espèce ovine imposent son utilisation dans le cadre de stations expérimentales 
avant d'envisager sa diffusion sur le terrain. Cette stratégie permettrait l'élaboration d'une banque d'embryons qui seraient par la suite transférés en vue de la mise en place d'une sélection massale et la préservation voire l'amélioration du patrimoine ovin algérien.

\section{Remerciements}

Les auteurs remercient Messieurs Gérard Baril de l'Institut national de recherche agronomique de Tours, France, et Jean-François Beckers de la Faculté de médecine vétérinaire de Liège, Belgique, pour leurs contribution et collaboration permanentes.

\section{REFERENCES}

Ammoun I., Encinas T., Veiga-Lopez A., Ros J.M., Contreras I., Gonzalez-Añover P., Cocero M.J., et al., 2006. Effects of breed on kinetics of ovine FSH and ovarian response in superovulated sheep. Theriogenology, 66 (4): 896-905, doi: 10.1016/j. theriogenology.2006.02.024

Bari F., Khalid M., Haresign W., Murray A., Merrell B., 2003. Factors affecting the survival of embryos after transfer within a MOET program. Theriogenology, $\mathbf{5 9}$ (5-6): 1265-1275, doi: 10.1016/S0093-691X(02)01162-7

Baril G., Brebion P., Chesne P., 1993. Manuel de formation pratique pour la transplantation embryonnaire chez la brebis et la chèvre. FAO, Rome, Italie, 183 p. (Production et santé animales ; 115)

Baril G., Guignot F., 2010. Production d'embryons in vivo et transfert chez les petits ruminants : synthèse des applications / résultats selon les races. Rencontres Rech. Rumin., 17 : 153-158

Boscos C., Vainas E., Kouskoura T.H., Vafiadis D., Dellis S., 1997. Superovulatory response of Chios and Friesian ewes to two FSH-P dose levels. Reprod. Domest. Anim., 32 (4): 195-198, doi: 10.1111/j.1439-0531.1997.tb01281.x

Buckrell B.C., Gartley C.I., Mehren KG., Goodrowe K.L., 1990. Superovulation in Dall's sheep (Ovis dalli dalli). Theriogenology, 33 (1): 201, doi: 10.1016/0093-691X(90)90625-4

Chagas e Silva J., Lopes da costa L., Cidadão R., Robalosilva J., 2003. Plasma progesterone profiles, ovulation rate, donor embryo yield and recipient embryo survival in native Salvia sheep in the fall and spring breeding seasons. Theriogenology, 60 (3): 521-532, doi: 10.1016/S0093691X(03)00045-1

Cognié Y., 1999. State of the art in sheep-goat embryo transfer. Theriogenology, 51 (1): 105-116, doi: 10.1016/S0093-691X(98)00235-0

Cognié Y., Baril G., Poulin N., Mermillod P., 2003. Current status of embryo technologies in sheep and goats. Theriogenology, 59 (1): 171-188, doi: 10.1016/S0093-691X(02)01270-0

D'Alessandro AG.D., Martemucci G., 2004. Efficiency of superovulatory treatment with $\mathrm{FSH}-\mathrm{p}$ for in vivo embryo production in dairy ewes: multiple versus single dose regimen. J. Anim. Vet. Adv., 3 (6): 388-393

D'Alessandro A.G.D., Martemucci G., 2016. Superovulatory response to gonadotrophin $\mathrm{FSH} / \mathrm{LH}$ treatment and effect of progestin supplement to recipients on survival of transferred vitrified embryos in goats. Theriogenology, 85 (2): 296-301, doi: 10.1016/j.theriogenology.2015.09.038

D'Alessandro A.G.D., Martemucci G., Taibi L., 2005. How the FSH/LH ratio and dose numbers in the $\mathrm{p}-\mathrm{FSH}$ administration treatment regimen, and insemination schedule affect superovulatory response in ewes. Theriogenology, 63 (6): 17641774, doi: 10.1016/j.theriogenology.2004.08.002

D'Alessandro A.G.D., Martemucci G., Toteda F., Gambacorta M., Manchis A., 1996. Superovulation and embryo production in ewes using a commercial pFSH. Small. Rumin. Res., 19 (3): 255261, doi: 10.1016/09214488(95)00765-2

Djaout A., Afri-Bouzebda F., Chekal F., El-Bouyahiaoui R., Rabhi A., Boubekeur A., Benidir M., et al., 2017. Etat de la biodiversité des "races » ovines algériennes. Gen. Biodiv. J., 1 (1) : 1-17

Dufour J.J., Cognié Y., Mermillod P., Mariana J.C., Romain R.F., 2000. Effects of the Booroola fec gene on ovarian follicular populations in superovulated Romanov ewes pretreated with a GnRH antagonist. J. Reprod. Fertil., 118 (2): 85 -94, doi: 10.1530/reprod/118.1.85
Feliachi K., Kerboua M., Abdelfettah M., Ouakli K., Selhab F., Boudjakdji A., Takoucht A., et al., 2003. Rapport national sur les ressources génétiques animales. Ministère de l'Agriculture et du Développement rural, Commission nationale AnGR, Algérie, $46 \mathrm{p}$.

Fierro S., Gil J., Viñoles C., Olivera-Muzante J., 2013. The use of prostaglandins in controlling estrous cycle of the ewe: A review. Theriogenology, 79 (3): 399408, doi: 10.1016/j.theriogenology.2012.10.022

Gibbons A., Cueto M., 2011. Training manual for embryo transfer in sheep and goats. INTA-EEA, San Carlos de Bariloche, Argentine, 35 p. (Comunicación técnica PA; 559)

Gonzalez F., Calero P., Beckers J.F., 2001. Induction of superovulation in domestic ruminants. In: Biotechnology in animal husbandry (Eds. Renaville R., Burny A.). Kluwer Academic, Netherlands, 209-223

Gonzalez-Bulnes A., Baird D.T., Campbell B.K., Cocero M.J., Garcia-Garcia R.M., Inskeep E.K., Lopez-Sebastian A., et al., 2004a. Multiple factors affecting the efficiency of multiple ovulation and embryo transfer in sheep and goats. J. Reprod. Fertil., 16 (6): 421-435, doi: 10.10371/RD04033

Gonzalez-Bulnes A., Garcia-Garcia R.M., Souza C.J.H., Santiago-Moreno J., Lopez-Sebastian A., Cocero M.J., Baird D.T., 2002. Patterns of follicular growth in superovulated ewes and influence on endocrine and ovarian response. Reprod. Domest. Anim., 37 (6): 357-361, doi: 10.1046/j.14390531.2002.00385.x

Gonzalez-Bulnes A., Santiago-Moreno J., Garcia-Garcia R.M., Souza C.J.H., Lopez-Sebastian A., Mcneilly A.S., 2004b. Effect of GnRH antagonist treatment on gonadotrophin secretion, follicular development and inhibin A secretion in goats. Theriogenology, 61 (5): 977-985, doi: 10.1016/j. theriogenology.2003.07.003

Guignot F., Baril G., Dupont F., Cognié Y., Folch J., Alabart J.L., Poulin N., et al., 2009. Determination of sex and scrapie resistance genotype in preimplantation ovine embryos. Mol. Reprod. Dev., 76 (2): 183-90, doi: 10.1002/mrd.20940

Institut technique des élevages, 2012. Infos élevages. Bull. trimestriel $n^{\circ} 1$. ITELV, Alger, Algérie, $4 \mathrm{p}$.

Kanitz W., Beckers J.F., Schneider F., Kanitz E., Leiding C., Nohner H.P., Pohland R., 2002. Superovulation in cattle: practical aspects of gonadotropin treatment and insemination. Reprod. Nutr. Dev., 42 (6): 587-599, doi: 10.1051/rnd : 2002045

Katanya J.F., Pawel M.B., King W.A., 2009. Relationship between circulating concentrations of ovarian steroids and the superovulatory responses in anestrous ewes following a multiple-dose pFSH regimen. Small Rumin. Res., 82 (2-3): 144-148, doi: 10.1016/j.smallrumres.2009.01.014

Lopes-Junior E.S., Maia E.L., Paula N.R., Teixeira D.I.A., Villarreal A.B.S., Rondina D., Freitas V.J.F., 2006. Effect of age of donor on embryo production in Morada Nova (white variety) ewes participating in a conservation programme in Brazil. Trop. Anim. Health Prod., 38 (7-8): 555-561, doi: 10.1007/s11250-006-4344-1

Maciel G.S., Rodriguez M.G.K., Da Silva P.D.A., Nociti R.P., Santos V.J.C., Uscategui R.A.R., Feliciano M.A.R., et al., 2017. Ovarian superstimulation treatment for multiple ovulation and embryo transfer programs in sheep. Investigação, 16 (8): 30-36, doi: 10.26843/investigacao.v16i8.1888

Martemucci G., Toteda F., Facciolongo A.M., D'Alessondro A.G.D., Gambacorta M., 1997. Ovarian response serum oestradiol-17B concentration and embryo yielded in anoestrus ewes treated with gonadotrophin pituitary extracts. Zoot. Nutr. Anim., 23: 81-88

Moula N., 2018. Caractérisation de la race ovine algérienne Tazegzawth. Tropicultura., 36 (1) : 43-53

Oliveira M.E.F., Felicianoa M.A.R., D'Amatoa C.C., Oliveiraa L.G., Bicudob S.D., Fonsecac J.F., Vicentea W.R.R., et al., 2014. Correlations between ovarian follicular blood flow and superovulatory responses in ewes. Anim. Reprod. Sci., 144 (1-2): 30-37, doi: 10.1016/j.anireprosci.2013.10.012

Picazo R.A., Cocero M.J., Barragán M.L., López-Sebastián A., 1996. Effects of $\mathrm{LH}$ administration at the end of an $\mathrm{FSH}$ superovulatory regimen on ovulation rate and embryo production in three breeds of sheep. Theriogenology, 45 (5): 1065-1073, doi: 10.1016/0093-691X(96)00033-7

Quan F., Zhang Z., An Z., Hua S., Zhao X., Zhang Y., 2011. Multiple factors affecting superovulation in Poll Dorset in China. Reprod. Domest. Anim., 46 (1): 39-44, doi: 10.1111/j.1439-0531.2009.01551.x

Rahman M.R., Rahman M.M., Wan Khadijah W.E., Abdullah R.B., 2014. Follicle stimulating hormone (FSH) dosage based on body weight enhances ovulatory responses and subsequent embryo production in goats. Asian Australas. J. Anim. Sci., 27 (9): 1270-1275, doi: 10.5713/ajas.2013.13786 
Rebolledo A.D., Manzanero G.V., Romero A.A., Franco J.Q., Rodriguez J.B., Lorca J.R., Ugalde J.R., 2017. Follicular population at the onset of a superovulatory treatment and ovarian response in hair ewes. Romanian Biotechnol. Lett., 22 (2): 12427-12431

Robertson I., Nelson R., 1999. Certification and identification of embryos. In: Procedural guide and general information for the use of embryo transfer technology, emphasizing sanitary precautions, 3rd Edn. International Embryo Transfer Society, Champaign, IL, USA, 109-122

Torres S., Cognié Y., 1984. Superovulation and egg transfer in the ewe. Reprod. Nutr. Dev., 24 (5A): 623-631, doi: 10.1051/rnd:19840510

\section{Summary}

Gharbi I., Dechicha A.S., Ferrouk M., Baazize-Ammi D., Kebbal S., Guetarni D. Effects of the gonadotropin dose on estrus and embryo production in Hamra and Ouled Djellal ewes

The application of superovulation and embryo production techniques is essential for the conservation of sheep genetic resources. The objective of this study was to evaluate the effect of two doses of porcine follicle-stimulating hormone $(\mathrm{pFSH})$ on the onset and duration of estrus, the ovarian response and the production of embryos in sheep of Ouled Djellal (OD) and Hamra $(H)$ breeds. OD ewes $(n=15)$ and H ewes $(n=14)$ were synchronized by the insertion of vaginal sponges impregnated with $40 \mathrm{mg}$ of fluorogestone acetate (FGA), and superovulated by the administration of 16 or $20 \mathrm{AU}$ of pFSH during the last three days of progestagen treatment. On day seven after estrus and natural mating the embryos were collected by laparotomy. In OD ewes treated with $20 \mathrm{AU}$ the onset of estrus occurred earlier than in those treated with $16 \mathrm{AU}(22.1 \pm 4.5$ vs $25.0 \pm 3.5, \mathrm{p}<0.05)$, and the duration of estrus was longer $(40.0 \pm 7.1$ vs $34.0 \pm 6.6, \mathrm{p}<0.05)$. In $\mathrm{H}$ ewes, the increase in the pFSH dose from $16 \mathrm{AU}$ to $20 \mathrm{AU}$ induced a decrease in embryo production: collection rate 72.2 vs. $47.1, \mathrm{p}<0.001$; number of structures collected $5.9 \pm 3.4$ vs $3.6 \pm 2.1, \mathrm{p}<0.05$; number of embryos harvested $5.1 \pm 2.6$ vs $3.2 \pm 1.9, p<0.05$; 1 -and-2-grade embryos $4.3 \pm 2.4$ vs $2.1 \pm 1.7, p<0.01$. On the other hand, in OD ewes treated with $20 \mathrm{AU}$ the ovulation rate and embryo production were significantly improved: number of corpora lutea $6.9 \pm 3.9$ vs $10.4 \pm 5.4, p<0.05$; number of embryos harvested $3.2 \pm 2.0$ vs $5.1 \pm 2.3, \mathrm{p}<0.05 ; 1$ - and 2 -grade embryos $2.2 \pm 1.6$ vs $4.3 \pm 1.7, \mathrm{p}<0.05$. The results indicate that the ovulatory response, estrus, and embryo production are influenced by the dose of $\mathrm{pFSH}$ and the breed.

Keywords: sheep, Ouled Djellal ewe, Hamra ewe, superovulation, $\mathrm{pFSH}$, animal embryo, Algeria
Veiga-Lopez A., Gonzalez-Bulnes A., Tresguerres J.A.F., Dominguez V., Ariznavarreta C., Cocero M.J., 2006. Causes, characteristics and consequences of anovulatory follicles in superovulated sheep. Domest. Anim. Endoc., 30 (2): 76-87, doi: 10.1016/j.domaniend.2005.06.001

Vivanco H.M., Greaney K.B., Varela H., 1994. Explaining the variability in superovulatory responses and yield of transferable embryos in sheep embryo transfer. Theriogenology, 41 (1): 329, doi: 10.1016/S0093-691X(05)80239-0

Vray M., Girault D., Hoog-Labouret N., Porcher R., Thalabard J.-C., 2004. Méthodologie des essais cliniques de petits effectifs. Thérapie, 59 (3) : 273 279, doi : 10.2515/therapie:2004054

\section{Resumen}

Gharbi I., Dechicha A.S., Ferrouk M., Baazize-Ammi D., Kebbal S., Guetarni D. Efecto de la dosis de gonadotropina sobre el estro y la producción del embrión en ovejas Hamra y Ouled Djellal

La aplicación de técnicas de superovulación y producción de embriones es esencial para la conservación de los recursos genéticos de las ovejas. El objetivo de este estudio fue evaluar el efecto de dos dosis de hormona estimulante del folículo porcino $(\mathrm{pFSH})$ sobre la aparición y la duración del estro, la respuesta ovárica y la producción de embriones en ovejas de razas Ouled Djellal (OD) y Hamra $(\mathrm{H})$. Las ovejas OD $(\mathrm{n}=15)$ y $\mathrm{H}(\mathrm{n}=14)$ se sincronizaron mediante la inserción de esponjas vaginales impregnadas con $40 \mathrm{mg}$ de acetato de fluorogestone (FGA), y superovuladas mediante la administración de 16 o 20 UA de pFSH durante los últimos tres días de tratamiento con progestágenos. Al día siete post estro y apareamiento natural, se recolectaron los embriones mediante laparotomía. En ovejas OD tratadas con $20 \mathrm{UA}$, el inicio del estro ocurrió antes que en aquellas tratadas con $16 \cup \mathrm{A}(22,1 \pm 4,5$ vs $25,0 \pm 3,5$, $\mathrm{p}<0,05)$, la duracion del estro fue mas prolongada $(40,0 \pm$ $7,1$ vs $34,0 \pm 6,6, p<0,05)$. En las ovejas $\mathrm{H}$, el aumento en la dosis de pFSH de 16 UA a $20 \mathrm{UA}$ indujo una disminución en la producción de embriones: tasa de recolección 72,2 vs $47,1, \mathrm{p}<0,001$; número de estructuras recogidas 5,9 $\pm 3,4$ vs $3,6 \pm 2,1, p<0,05$; numero de embriones recolectados $5,1 \pm$ 2,6 vs $3,2 \pm 1,9, p<0,05$; embriones de grado 1 y $2,4,3 \pm 2,4$ vs $2,1 \pm 1,7, p<0,01$. Por otro lado, en ovejas OD tratadas con 20 UA, la tasa de ovulación y la producción de embriones mejoraron significativamente: número de cuerpos lúteos 6,9 $\pm 3,9$ vs $10,4 \pm 5,4, p<0,05$; número de embriones recolectados $3,2 \pm 2,0$ vs $5,1 \pm 2,3, p<0,05$; embriones de grado 1 y 2 , $2,2 \pm 1,6$ vs $4,3 \pm 1,7, p<0,05$. Los resultados indican que la dosis de $\mathrm{pFSH}$ y la raza influyen en la respuesta ovulatoria, el estro y la producción de embriones.

Palabras clave: ovino, oveja Ouled Djellal, oveja Hamra, superovulación, pFSH, embrión animal, Argelia 
